Bull. Korean Math. Soc. 46 (2009), No. 5, pp. 1019-1029

DOI 10.4134/BKMS.2009.46.5.1019

\title{
THE DISJOINT CURVE PROPERTY AND BRIDGE SURFACES
}

\author{
Sungbok Hong And Heoung Sook Kim
}

\begin{abstract}
We show that every bridge surface of certain types of $(1,1)$ prime knot has the disjoint curve property. Also we determine when a bridge surface of a pretzel knot of type $(-2,3, n)$ has the disjoint curve property.
\end{abstract}

\section{Introduction}

Let $M$ denote a compact orientable 3 -manifold and let $\left(W_{1}, W_{2} ; H\right)$ be a genus $g$ Heegaard splitting of $M$. In 1960s, W. Haken [4] introduced a condition of Heegaard splittings which is called the reducibility. A splitting $\left(W_{1}, W_{2} ; H\right)$ is said to be reducible if there are essential disks $D_{i} \subset W_{i}(i=1,2)$ with $\partial D_{1}=\partial D_{2}$. Otherwise, $\left(W_{1}, W_{2} ; H\right)$ is said to be irreducible. He proved that all splittings of a reducible manifold are themselves reducible. Strongly irreducibility was introduced by A. Casson and McA. Gordon [3] as a generalization of irreducibility. A splitting $\left(W_{1}, W_{2} ; H\right)$ is said to be weakly reducible if there are essential disks $D_{i} \subset W_{i}(i=1,2)$ with $\partial D_{1} \cap \partial D_{2}=\emptyset$. Otherwise, $\left(W_{1}, W_{2} ; H\right)$ is said to be strongly irreducible. They showed that all splittings of a non-Haken manifold are either reducible or strongly irreducible. A. Thompson [17] introduced the notion of disjoint curve property as a further generalization of reducibility. A splitting $\left(W_{1}, W_{2} ; H\right)$ admits the disjoint curve property if there are essential disks $D_{i} \subset W_{i}(i=1,2)$ and an essential loop $c \subset H$ with $\left(\partial D_{1} \cup \partial D_{2}\right) \cap c=\emptyset$. A splitting is full if it does not have the disjoint curve property. A. Thompson proved that all splittings of a toroidal 3-manifold have the disjoint curve property in [17]. And J. Hempel has shown that each splitting of a Seifert fibred space has the disjoint curve property using the classification of splittings of Seifert fibered spaces in [8]. Thus, in any 3-manifold which is reducible, toroidal, or a Seifert fibred space, all Heegaard splittings have the disjoint curve property.

Received August 27, 2008; Revised January 19, 2009

2000 Mathematics Subject Classification. Primary 57M99; Secondary 57M27, 57N10.

Key words and phrases. disjoint curve property, bridge surface, (1,1)-knot, Heegaard splitting.

Supported by Korea University Grant.

(C)2009 The Korean Mathematical Society 
However, it is certainly not the case that all splittings of all manifolds have the disjoint curve property. J. Hempel in [8] adapts an argument of T. Kobayashi [11] to produce examples of splittings which are full and are in fact arbitrarily far from having the disjoint curve property.

In this paper, we show that every bridge surface of certain types of $(1,1)$ prime knot has the disjoint curve property. T. Saito has shown that a bridge surface of $(1,1)$-hyperbolic knot is full, except for certain type of knots in [15]. Also we determine that every bridge surface of the pretzel knot of type $(-2,3, n)$ has the disjoint curve property.

Acknowledgement. We would like to thank to the referee for useful comments and suggestions.

\section{Preliminaries}

In this section, we recall standard notation and review several notions from the theory of $g$-genus $n$-bridge Heegaard splittings. We would like to refer [5] for more detail about relationship between definitions.

Let $W$ be a 3-manifold with non-empty boundary $\partial W$ and $K=\left\{k_{1}, k_{2}, \ldots\right.$, $\left.k_{n}\right\}$ be a set of disjoint arcs properly embedded in $W$, that is, $k_{i} \cap \partial W=\partial k_{i}$ for every $1 \leq i \leq n$.

Definition 2.1. $K$ is trivial in $(W, K)$ if there is a set $\left\{D_{1}, \ldots, D_{n}\right\}$ of disjoint discs embedded in $W$ such that $D_{i} \cap\left(\cup k_{i}\right)=\partial D_{i} \cap k_{i}=k_{i}$ and so that $D_{i} \cap \partial W$ is the $\operatorname{arc} \operatorname{cl}\left(\partial D_{i}-k_{i}\right)$. We call $D_{i}$ a cancelling disc of $k_{i}$.

When $W$ is a ball and $K$ is trivial, the pair $(W, K)$ is called a trivial $n$-string tangle.

Let $T$ be a properly embedded 1-manifold in $W$, that is, $T \cap \partial W=\partial T$ and let $F$ be a 2-manifold properly embedded in $W$. Suppose that $F$ is transverse to $T$. In particular $\partial F \cap T=\emptyset$.

Definition 2.2. $F$ is $T$-compressible if there is a disc $D$ embedded in $W$ such that $D$ is disjoint from $T$, that is $D \cap F=\partial D$ and that $\partial D$ dose not bound a disc disjoint from $T$ on $F$. Such $D$ is called a $T$-compressing disc of $F$. We call $F$ a $T$-incompressible if it is not $T$-compressible.

Definition 2.3. A 2-manifold $F$ is meridionally compressible in $(W, T)$ if there is a disc $D$ embedded in $W$ such that int $D$ intersects $T$ transversely in a single point, that $D \cap F=\partial D \cap(F-T)=\partial D$ and that $\partial D$ in $F$ does not bound a disc whose interior intersects $T$ transversely in a single point. Such $D$ is called a meridionally compressing disc of $F . F$ is meridionally incompressible if it is not meridionally compressible.

We define a $T$-compressible 2-submanifold and a meridionally compressible 2 -submanifold of $\partial W$ similarly. Assume that either $F$ is a 2-manifold properly embedded in $W$ such that $F$ is transverse to $T$, or $F$ is a 2 -submanifold of $\partial W$ 
with $\partial F \cap T=\emptyset$. A simple loop $l$ on $F$ is said to be $T$-essential if it is disjoint from $T$ and if it does not bound a disc which intersects $T$ transversely in zero or one point.

Let $M$ be a closed orientable 3 -manifold and $L$ a link in $M$. Let $H$ be a genus $g$ Heegaard splitting surface of $M$, that is, $H$ divides $M$ into two handlebodies $W_{1}$ and $W_{2}$ of genus $g$. Suppose that $H$ is transverse to $L$.

Definition 2.4. $H$ is a $g$-genus $n$-bridge splitting, $(g, n)$-splitting, of $(M, L)$ if $L$ intersects $W_{i}$ in a set of trivial $n$ arcs for $i=1,2$.

A link $L$ is called a g-genus $n$-bridge link, $(g, n)$-link, if it admits a $g$-genus $n$-bridge splitting. A link in $S^{3}$ is simply called an $n$-bridge link in $S^{3}$ if it has a 0 -genus $n$-bridge spitting.

Let $K$ be a knot in a closed 3-manifold $M$. Let $(M, K)=\left(W_{1}, k_{1}\right) \cup_{H}$ $\left(W_{2}, k_{2}\right)$ be a bridge splitting of a 3 -manifold $(M, K)$.

Definition 2.5. $H$ is $K$-reducible if $W_{1}$ and $W_{2}$ contain $K$-compressing or meridionally compressing discs $D_{1}$ and $D_{2}$ of $H$ respectively such that $\partial D_{1}=$ $\partial D_{2}$ in $H . H$ is $K$-irreducible if it is not $K$-reducible.

Note that if $H$ is $K$-reducible, then $K$ is the trivial knot bounding a disc composed of two cancelling discs as shown in [6].

Definition 2.6. $H$ is weakly $K$-reducible if $W_{1}$ and $W_{2}$ contain $K$-compressing or meridionally compressing discs $D_{1}$ and $D_{2}$ of $H$ respectively such that $\partial D_{1} \cap$ $\partial D_{2}=\emptyset . H$ is strongly $K$-irreducible if it is not weakly $K$-reducible.

Definition 2.7. $H$ has the disjoint curve property if there exist essential simple closed curves $c, \partial D_{1}$, and $\partial D_{2}$ on $H$ such that $c \cap\left(\partial D_{1} \cup \partial D_{2}\right)=\emptyset$ and $D_{1}$ and $D_{2}$ are $K$-compressing or meridionally compressing discs in $W_{1}$ and $W_{2}$ respectively.

By $E(K)$, we mean the exterior of $K$ in $M$, i.e., $E(K)=\operatorname{cl}(M-N(K))$, where $N(K)$ is a regular neighborhood of $K$ in $M$.

Definition 2.8. (1) $K$ is a trivial knot if it bounds a disc imbedded in $M$.

(2) $K$ is a core knot if $K$ is non-trivial and $M$ admits a genus one Heegaard splitting $\left(V_{1}, V_{2} ; P\right)$ such that $K$ is isotopic to the core of $V_{i}$ for $i=1$ or 2 .

(3) $K$ is a 2-bridge knot if there is a genus zero Heegaard splitting $\left(B_{1}, B_{2}\right.$ : $\left.P_{0}\right)$ of $S^{3}$ such that $\left(B_{i}, B_{i} \cap K\right)(i=1,2)$ is a 2 -string trivial tangle.

(4) $K$ is split if $M$ contains a sphere $S$ which decomposes $M$ into a punctured lens space and a ball containing $K$ in its interior. This sphere $S$ is called a splitting sphere.

(5) $K$ is a composite knot if $M$ contains a 2-sphere $S$ which intersects $K$ transversely in 2 points and $S \cap E(K)$ is $\partial$-incompressible in $E(K)$. We call this 2 -sphere $S$ a decomposing sphere. A knot is said to be prime if it is not composite. 
It is proved in [7] that if $H$ is weakly $K$-reducible, then $K$ is the trivial knot or a 2-bridge knot when $M=S^{3}$, and $K$ is a core knot or a composite knot of a core knot and a 2-bridge knot when $M$ is a lens space.

Definition 2.9. A knot $K$ is called a torus knot if $K$ is isotopic to a simple loop on a genus one Heegaard surface of $M$ and is not a core knot.

The torus knot $T_{p, q}$ of type $(p, q)$ is the knot which wraps around the standard solid torus $p$ times in the longitudinal direction, and $q$ times in the meridional direction.

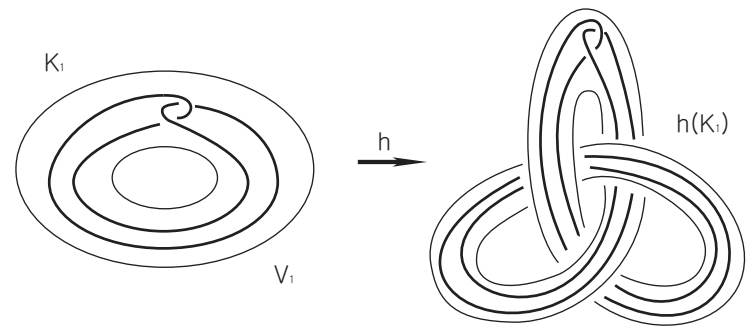

Figure 1. Satellite knot

Let $V_{1}$ be an unknotted solid torus in $S^{3}$ and $K_{1}$ be a knot in $V_{1}$. Let $V_{2}$ be a tubular neighborhood of a non-trivial knot $K_{2}$. For a homeomorphism $h$ : $V_{1} \rightarrow V_{2}$, the knot $K=h\left(K_{1}\right)$ is called a satellite knot and $K_{2}$ its companion.

Definition 2.10. A knot $K$ is said to be satellite if $E(K)$ contains an incompressible torus $T$ which is not parallel to $\partial E(K)$.

Definition 2.11. A hyperbolic knot is a knot whose complement can be endowed with a metric of constant curvature -1 .

The seminal work of W. Thurston demonstrates that every knot in $S^{3}$ is either a torus knot, a satellite knot or a hyperbolic knot. These three categories are mutually exclusive.

\section{Heegaard splitting}

In Section 3, we may consider 3-manifolds with nonempty boundaries such as knot complements. In those cases, we use the notion of Heegaard splitting of compression body.

J. Hempel has shown that if a closed orientable 3-manifold $M$ is reducible, Seifert fibered or toroidal, then any splitting of $M$ has the disjoint curve property in [8].

J. Hempel's result is generalized to the Proposition 3.1 by T. Saito [16]. He introduced a notion called the disjoint $(A, D)$-pair property. Here, a Heegaard splitting $\left(W_{1}, W_{2} ; H\right)$ admits the disjoint $(A, D)$-pair property if there are an essential annulus $A_{i}$ normally embedded in $W_{i}$ and an essential disk $D_{j}$ in $W_{j}$, $((i, j)=(1,2)$ or $(2,1))$ such that $\partial A_{i} \cap \partial D_{j}=\emptyset$. 
Proposition 3.1 ([16]). Let $M$ be a compact orientable 3-manifold. If $M$ is reducible, Seifert fibered or toroidal, then any genus $g \geq 2$ Heegaard splitting of $M$ admits the disjoint $(A, D)$-pair property. Moreover, if a Heegaard splitting admits the disjoint $(A, D)$-pair property, then it admits the disjoint curve property.

We can deduce the following corollary from Proposition 3.1.

Corollary 3.2. If $K$ is a torus knot in $S^{3}$, then every genus $g \geq 2$ Heegaard splitting of $E(K)$ has the disjoint curve property.

Proof. Since $K$ is a torus knot, $E(K)$ is a Seifert fibered manifold. Let $E(K)=$ $W_{1} \cup_{H} W_{2}$ for $g \geq 2$. By Proposition 3.1, $H$ has the disjoint curve property.

A 3-manifold is toroidal if it contains an essential torus, namely an incompressible torus which is not parallel to a boundary component.

Corollary 3.3. If $K$ is a satellite knot in $S^{3}$, then every genus $g \geq 2$ Heegaard splitting of $E(K)$ has the disjoint curve property.

Proof. Since $K$ is a satellite knot, $E(K)$ is toroidal. Let $E(K)=W_{1} \cup_{H} W_{2}$ for $g \geq 2$. By Proposition 3.1, $H$ has the disjoint curve property.

\section{Bridge surface}

The next two propositions guarantee the existence of cancelling discs satisfying certain conditions for a Heegaard surface which is in $(1,1)$ position with respect to the given knot.

Proposition 4.1 ([13, Theorem 3]). Let $L$ be a lens space and $K$ a 1-bridge knot in $L$, and let $\left(W_{1}, W_{2} ; H\right)$ be a Heegaard splitting of genus one of $L$ which gives a 1-bridge representation of $K$ i.e., $\alpha_{i}=W_{i} \cap K$ is a single trivial arc in $W_{i}(i=1,2)$.

Suppose that $K$ is a non-trivial torus knot and is not a core of $L$. Then for $i=1,2$, there exists a disk $\triangle_{i}$ in $W_{i}$ such that $\partial W_{i} \cap \triangle_{i}=\beta_{i}$ is an arc in $\partial W_{i}, \partial \triangle_{i}=\alpha_{i} \cup \beta_{i}$ and $\beta_{1} \cap \beta_{2}=\partial \beta_{1}=\partial \beta_{2}$.

Proposition 4.2 ([7, Theorem III]). Let $M$ be the $S^{3}$ or a lens space (not homeomorphic to $\left.S^{2} \times S^{1}\right)$. Let $H$ be a genus 1 Heegaard surface of $M$. This surface $H$ divides $M$ into two solid tori $W_{1}$ and $W_{2}$. Suppose a knot $K$ is in 1-genus 1-bridge position with respect to $H$ and $H$ is neither $K$-reducible nor weakly $K$-reducible.

If $K$ is a satellite knot, then there is an annulus $Z$ on $H$ such that there is a cancelling disc $C_{i}$ of $t_{i}$ (trivial arc in $\left.V_{i}\right)$ with $\left(\partial C_{i} \cap H\right) \subset Z$ for $i=1,2$. Moreover, the incompressible torus is isotopic to $\partial N\left(C_{1} \cup Z \cup C_{2}\right)$ in $E(K)$.

Using Propositions 4.1 and 4.2, we can deduce the disjoint curve property for bridge surfaces of non trivial torus knots and satellite knots. 
Theorem 4.3. Let $M$ be the $S^{3}$ or a lens space and $K$ be a 1-bridge knot in $M$. If $K$ is a non-trivial torus knot in $M$, then the bridge surface $H$ has the disjoint curve property.

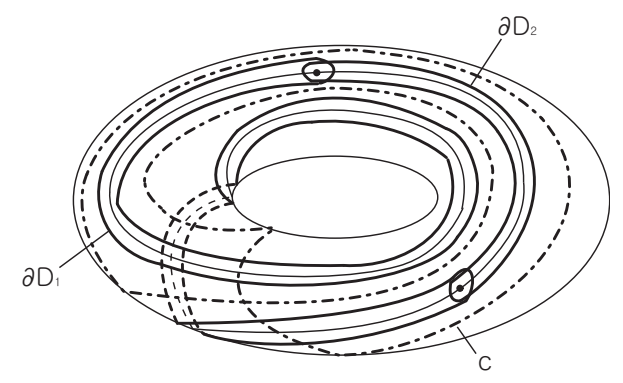

FIGURE 2. Essential curves satisfying the disjoint curve property for a torus knot

Proof. Any 1-bridge representation of a torus knot in a lens space is trivial. Let $(M, K)=\left(W_{1}, \alpha_{1}\right) \cup_{H}\left(W_{2}, \alpha_{2}\right)$ and $C_{i}$ be a cancelling disc of $\alpha_{i}$. By Proposition 4.1, $H \cap C_{i}=\beta_{i}$ is an arc in $H$. Then $A=N\left(\beta_{1} \cup \beta_{2}\right)$ is an annulus in $H$. Now the disc $D_{i}=\operatorname{cl}\left(\partial N\left(C_{i}, W_{i}\right)-H\right)$ is a properly embedded and $K$-compressing disc in $W_{i}$ for $i=1,2 . c l(H-\operatorname{int}(A))$ is an annulus in $H$. Moreover one can find an essential curve $c$ in $c l(H-\operatorname{int}(A))$ such that $\partial D_{1} \cap c=\emptyset$ and $\partial D_{2} \cap c=\emptyset$. Therefore $H$ has the disjoint curve property. We describe explicitly how to get essential curves satisfying the disjoint curve property for a torus knot in Figure 2.

Theorem 4.4. Let $M$ be the $S^{3}$ or a lens space (not homeomorphic to $S^{2} \times S^{1}$ ). Let $H$ be a genus 1 Heegaard surface of $M$. This surface $H$ divides $M$ into two solid tori $W_{1}$ and $W_{2}$. Suppose a knot $K$ is in 1-genus 1-bridge position with respect to $H$. If $K$ is a satellite knot, then $H$ has the disjoint curve property.

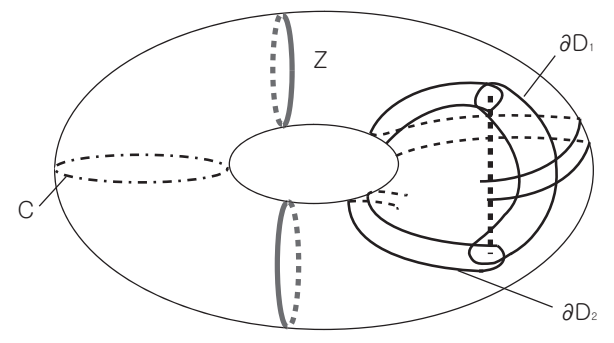

FiguRe 3 


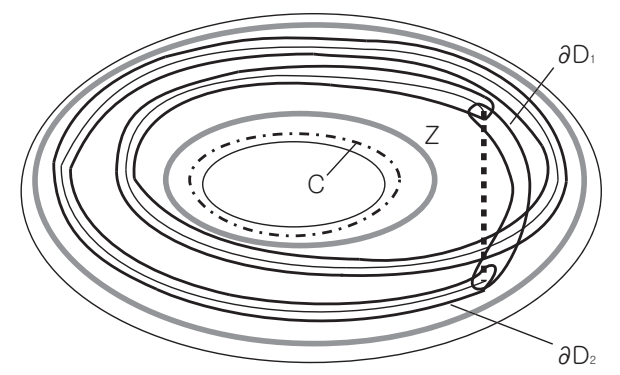

FiguRe 4

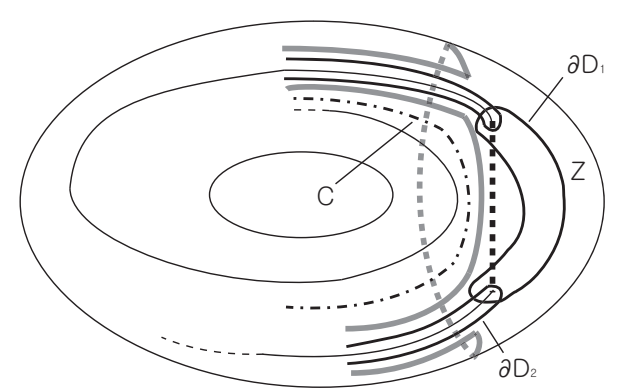

FiguRe 5

Proof. If $H$ is $K$-reducible, then $K$ is a trivial knot by [15]. If $H$ is weakly $K$-reducible, then $M$ is $S^{2} \times S^{1}$ and $K$ is a core knot by [15]. By assumption, we may assume that $H$ is neither $K$-reducible nor weakly $K$-reducible. Let $(M, K)=\left(W_{1}, t_{1}\right) \cup_{H}\left(W_{2}, t_{2}\right)$, and $C_{i}$ be a cancelling disc of $t_{i}$. By Proposition 4.2, there exists an annulus $Z$ in $H$ such that there is a cancelling disc $C_{i}$ of $t_{i}$ with $\left(\partial C_{i} \cap H\right) \subset Z$ for $i=1,2$. If we take $D_{i}=c l\left(\partial N\left(C_{i}, W_{i}\right)-H\right)$, then it is a properly embedded and $K$-compressing disc in $W_{i}$ for $i=1,2$. Because $c l(H-\operatorname{int}(Z))$ is an annulus, one can find an essential curve $c$ in $c l(H-\operatorname{int}(Z))$ such that $\partial D_{1} \cap c=\emptyset$ and $\partial D_{2} \cap c=\emptyset$. We describe explicitly how to get essential curves which satisfy the disjoint curve property for the case when $\partial C_{2} \cap H$ is meridinal in Figure 3, the case when $\partial C_{2} \cap H$ is logitudinal in Figure 4 and the case when $\partial C_{2} \cap H$ is neither meridinal nor logitudinal in Figure 5. The dotted line in each figure indicates the knot in a splitted solid torus. Therefore $H$ has the disjoint curve property.

Now we consider hyperbolic knots.

A knot $K$ in an orientable closed 3-manifold $M$ is called a $(1,1)$-knot if $(M, K)=\left(W_{1}, k_{1}\right) \cup_{H}\left(W_{2}, k_{2}\right)$. To define the distance of a $(1,1)$-splitting, we 
use the twice punctured torus $\Sigma=H-K$. For notation and definition, we refer to [15].

For $i=1$ or 2 , let $\mathcal{K}\left(W_{i}\right)$ be the maximal subcomplex of $C(\Sigma)$ consisting of simplexes $\left\langle\left[c_{0}\right],\left[c_{1}\right], \ldots,\left[c_{k}\right]\right\rangle$ such that an essential loop representing $\left[c_{j}\right]$ $(j=0,1, \ldots, k)$ bounds a disk in $W_{i}-k_{i}$.

Definition. We define the distance of a $(1,1)$-splitting $\left(W_{1}, W_{2} ; H\right)$ by $d\left(W_{1}, W_{2}\right)=\min \left\{d([x],[y]) \mid[x]:\right.$ a vertex in $\mathcal{K}\left(W_{1}\right),[y]:$ a vertex in $\left.\mathcal{K}\left(W_{2}\right)\right\}$.

For a pair $\alpha(\geq 4)$ and $\beta$ of co-prime integers and an element $\gamma \in \mathbb{Q} \cup\{1 / 0\}$, $K(\alpha, \beta ; \gamma)$ denotes the knot $K_{2}$ in $K_{1}(\gamma)$, where $K_{1} \cup K_{2}$ is the 2-bridge link of type $(\alpha, \beta)$ (cf. Chapter 10 of [14]) and $K_{1}(\gamma)$ is the manifold obtained by $\gamma$-surgery on $K_{1}$. By definition, every $K(\alpha, \beta ; \gamma)$ is a $(1,1)$-knot.

Proposition $4.5([15])$. Let $K$ be a $(1,1)$-knot in $M$. Suppose that $(M, K)$ is not equivalent to $K(\alpha, \beta ; \gamma)$ for any $\alpha, \beta$ and $\gamma$, and that the bridge index of $K$ is at least three if $M \cong S^{3}$. Then $K$ is a hyperbolic knot if and only if it has a $(1,1)$-splitting with distance $\geq 3$.

By Proposition 4.5, one can see that a $(1,1)$-knot is hyperbolic if a $(1,1)$ splitting does not have the disjoint curve property and a $(1,1)$-splitting with distance neither 0 nor 1 .

Let $L\left(p_{1}, p_{2}, \ldots, p_{n}\right)$ be an $n$-pretzel link in $S^{3}$ where $p_{i} \in \mathbb{Z}$ represents the number of half twists. In particular, if $n=3$, it is called a classical pretzel link, denoted by $L(p, q, r)$. For notation and definition for pretzel knots, we refer to [10].

If $n$ is odd, then an $n$-pretzel link $L\left(p_{1}, p_{2}, \ldots, p_{n}\right)$ is a knot if and only if none of two $p_{i}$ 's are even. If $n$ is even, then $L\left(p_{1}, p_{2}, \ldots, p_{n}\right)$ is a knot if and only if one of the $p_{i}$ 's is even. A pretzel knot is denoted by $K\left(p_{1}, p_{2}, \ldots, p_{n}\right)$.

A link $L$ is almost alternating if it is not alternating and there is a diagram $D_{L}$ of $L$ such that one crossing change makes the diagram alternating; we call $D_{L}$ an almost alternating diagram. By [10], classical pretzel links are prime and either alternating or almost alternating. W. Menasco has shown that prime alternating knots are either hyperbolic or torus knots [12]. It has been generalized by C. Adams that prime almost alternating knots are either hyperbolic or torus knots [2]. It is known that no satellite knot is an almost alternating knot [9]. By the following theorem [10], we can classify classical pretzel knots completely into hyperbolic or torus knots.

Proposition 4.6 ([10]). The following are the only nontrivial pretzel knots which are torus knots.

(1) $K(p, \pm 1, \mp 1)$ are unknots for all $p$.

(2) $K( \pm 1, \pm 1, \pm 1)$ are $(2, \pm 3)$ torus knots.

(3) $K( \pm 2, \mp 1, \pm r)$ are $(2, \pm r \mp 2)$ torus knots.

(4) $K(\mp 2, \pm 3, \pm 3), K(\mp 2, \pm 3, \pm 5)$ are $(3, \pm 4),(3, \pm 5)$ torus knots, respectively. 
Now we consider a pretzel knot of type $(-2,3, n)$. Every $K(-2,3, n)$ pretzel knot, $n \in \mathbb{Z}$, can be described by the diagram as in Figure 6 .

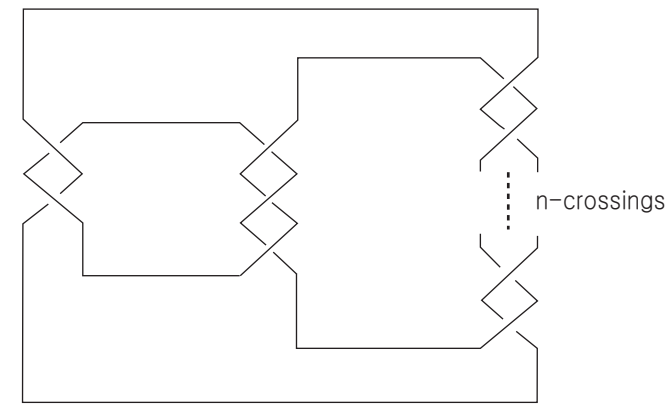

Figure 6. $(-2,3, n)$ pretzel knot diagram

Theorem 4.7. For pretzel knots of type $(-2,3, n)$, the following holds.

(1) If $n=1,3,5$, then the genus one 1-bridge surface which has the disjoint curve property.

(2) If $n \neq 1,3,5$, then there is a genus two 1-bridge surface which has the disjoint curve property.

Proof. If $n=1,3,5$, then $K(-2,3, n)$ is a nontrivial torus knot by Proposition 4.6. According to Theorem 4.3, genus one 1-bridge surface has the disjoint curve property.

If $n \neq 1,3,5$, then each of these knots has a hyperbolic structure on its complement. Each pretzel knot of type $K(-2,3, n)$ admits $(2,1)$-splitting as follows. The given knot can be realized as a curve on the surface of a double torus. $S^{3}$ has a genus two Heegaard splitting. Namely, $S^{3}$ can be thought of as two double tori whose boundaries have been identified. Actually, $S^{3}$ has a unique Heegaard splitting of each genus.
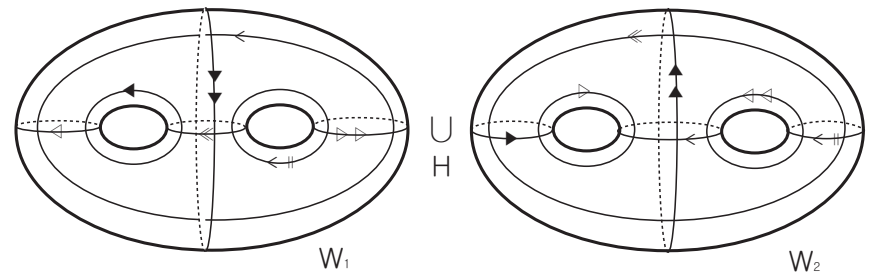

FiguRE 7. (2,1)-splitting

Let $\left(W_{1}, W_{2} ; H\right)$ be a Heegaard splitting of genus 2 of $S^{3}$ and the knot can be divided into two arcs namely, $K(-2,3, n)=K_{1} \cup K_{2}$. Let $t_{i}$ be a trivial arc 
in $W_{i}$ obtained from $K_{i}$ by pushing interior of $K_{i}$ into int $W_{i}$ for $i=1,2$. Then $(M, K)=\left(W_{1}, t_{1}\right) \cup_{H}\left(W_{2}, t_{2}\right)$ is a $(2,1)$-splitting, and $C_{i}$ is a cancelling disc of $t_{i}$ (see Figure 7 for details).

If we take $D_{i}=\operatorname{cl}\left(\partial N\left(C_{i}, W_{i}\right)-H\right)$, then it is a properly embedded $K$ compressing disc in $W_{i}$ for $i=1,2$.

Because $\partial\left(N\left(C_{1}, H\right) \cup N\left(C_{2}, H\right)\right)$ is an annulus in $H$, one can find an essential curve $c$ in $c l\left(H-\partial\left(N\left(C_{1}, H\right) \cup N\left(C_{2}, H\right)\right)\right)$ such that $\partial D_{1} \cap c=\emptyset$ and $\partial D_{2} \cap c=$ $\emptyset$. Therefore $H$ has the disjoint curve property. This completes the proof of Theorem 4.7.

Example. We now describe how to construct $(2,1)$-splitting and how to find 3 essential circles which satisfy the disjoint curve property for the $(-2,3,7)$ pretzel knot in Figure 8 and Figure 9.

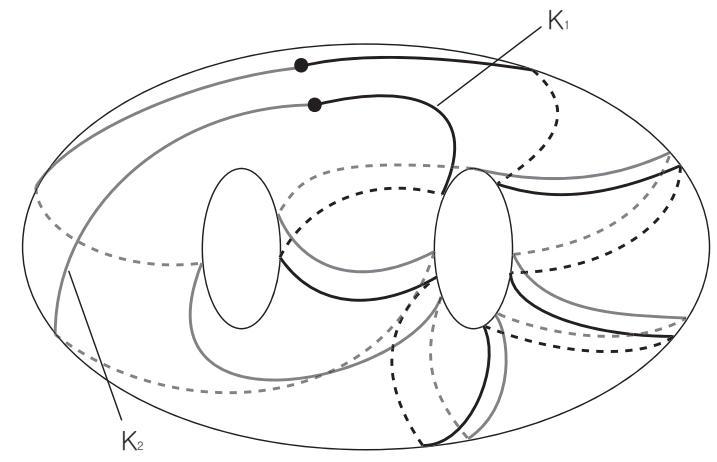

Figure 8. (2,1)-splitting for a $(-2,3,7)$ pretzel knot

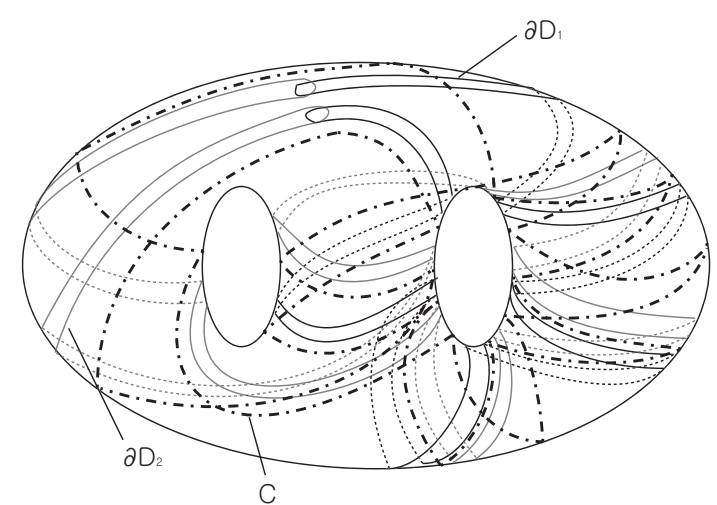

FiguRE 9. Essential curves satisfying the disjoint curve property for a $(-2,3,7)$ pretzel knot 


\section{References}

[1] C. Adams, The Knot Book, W. H. Freeman and Company, New York, 1994.

[2] C. Adams, J. Brock, J. Bugbee, and et al, Almost alternating links, Topology Appl. 46 (1992), no. 2, 151-165.

[3] A. J. Casson and C. McA. Gordon, Reducing Heegaard splittings, Topology Appl. 27 (1987), no. 3, 275-283.

[4] W. Haken, Studies in Modern Topology; Some results on surfaces in 3-manifolds, Prentice Hall, 1968.

[5] C. Hayashi, 1-genus 1-bridge splittings for knots, Osaka J. Math. 41 (2004), no. 2, 371-426.

[6] Genus one 1-bridge positions for the trivial knot and cabled knots, Math. Proc. Cambridge Philos. Soc. 125 (1999), no. 1, 53-65.

[7] _ Satellite knots in 1-genus 1-bridge positions, Osaka J. Math. 36 (1999), no. 3, 711-729.

[8] J. Hempel, 3-manifolds as viewed from the curve complex, Topology 40 (2001), no. 3, 631-657.

[9] J. Hoste, M. Thistlethwaite, and J. Weeks, The first 1,701,936 knots, Math. Intelligencer 20 (1998), no. 4, 33-48.

[10] D. Kim and E. Lee, Some invariants of pretzel links, arXiv:math.GT0704.1432v1, preprint.

[11] T. Kobayashi, Heights of simple loops and pseudo-Anosov homeomorphisms, Braids (Santa Cruz, CA, 1986), 327-338, Contemp. Math., 78, Amer. Math. Soc., Providence, RI, 1988.

[12] W. Menasco, Closed incompressible surfaces in alternating knot and link complements, Topology 23 (1984), no. 1, 37-44.

[13] K. Morimoto, On minimum genus Heegaard splittings of some orientable closed 3manifolds, Tokyo J. Math. 12 (1989), no. 2, 321-355.

[14] D. Rolfsen, Knots and Links, Publish or Perish, Inc., Houston, TX, 1990.

[15] T. Saito, Genus one 1-bridge knots as viewed from the curve complex, Osaka J. Math. 41 (2004), no. 2, 427-454.

[16] Disjoint pairs of annuli and disks for Heegaard splittings, J. Korean Math. Soc. 42 (2005), no. 4, 773-793.

[17] A. Thompson, The disjoint curve property and genus 2 manifolds, Topology Appl. 97 (1999), no. 3, 273-279.

SUNGBOK HONG

Department of Mathematics

Korea UnIVERSITY

SEOUL 136-713, KOREA

E-mail address: shong@korea.ac.kr

Heoung SoOK KIM

Department of Mathematics

Korea University

SEOUl 136-713, KoreA

E-mail address: heoung95@korea.ac.kr 